\title{
Narrativa y educación con perspectiva decolonial
}

\section{Narrative and education with decolonial perspective}

José Ignacio Rivas-Flores, ${ }^{*}$ María Jesús Márquez-García, ${ }^{* *}$ Analía Elizabeth Leite-Méndez, ${ }^{* * *}$ Pablo Cortés-González $* * * *$

Recibido: 31 de mayo de 2020 Aceptado: 14 de junio de 2020 Publicado: 30 de septiembre de 2020

To cite this article: Rivas-Flores, J. I., Márquez-García, M. J., Leite-Méndez, A. y Cortés-González, P. (2020). Narrativa y educación con perspectiva decolonial. Márgenes, Revista de Educación de la Universidad de Málaga, 1 (3), 46-62

DOI: https://doi.org/10.24310/mgnmar.v1i3.9495

\section{RESUMEN}

En este artículo planteamos la investigación narrativa en educación desde una posición “otra”, es decir, alejada de una perspectiva epistemológica interpretativa, hegemónica e influenciada por un pensamiento neoliberal gerencialista de la educación, que se sitúa en una posición utilitarista-asimétrica entre investigador/aparticipante. Esta posición jerárquica “subalterniza” a las personas vulnerables, especialmente de los colectivos marginados en la escuela y socialmente. Conformamos un recorrido epistemológico y metodológico en otro sentido, pensamos la investigación narrativa en educación en clave decolonial como un proceso ético-político, de relación, de diálogo y de co-interpretación horizontal, en el que co-participamos en un proceso de transformación y acción colectiva. Entendemos que la narrativa y la transformación, global y local, personal y colectiva, se articulan en un mismo escenario.

El giro decolonial plantea un doble desafío a la investigación narrativa, por una parte resignificar el conocimiento, las metodologías, el contexto académico y el sentido de la narrativa como proceso decolonizador; y por otro, nos sitúa en el terreno de la vida para co-construir, en el contexto social y educativo, espacios de saberes compartidos y de acción conjunta, con metodologías de carácter performativo que provoquen un debate social y educativo alternativo.

Palabras clave: transformación social; epistemologías; metodologías horizontales

\section{ABSTRACT}

In this paper, we show narrative research in education from a position "other". That is, a perspective that works away from an interpretive, hegemonic epistemological perspective, which is influenced by a neoliberal managerialist view of education, situated in a utilitarian-asymmetric position between researcher/participant. This

José Ignacio Rivas-Flores

https://orcid.org/0000-0001-5571-2011

Universidad de Málaga (España)

i_rivas@uma.es

María Jesús Márquez-García https://orcid.org/0000-0002-2220-3795

Universidad de Málaga (España)

mariajesusmarquez@uma.es
Analía Elizabeth Leite-Méndez

https://orcid.org/0000-0001-5064-999X

Universidad de Málaga (España)

aleite@uma.es

Pablo Cortés-González

https://orcid.org/0000-0002-9604-044X

Universidad de Málaga (España)

pcortes@uma.es 


\section{EST U D O S Y E N A Y O S}

hierarchical position "subordinates" vulnerable people, especially from marginalized groups at school and socially. We go around an epistemological and methodological journey in another sense. We think about narrative research in education in a decolonial key, as an ethical-political process, of relationship, dialogue and horizontal co-interpretation, in which we co-participate in a process of transformation and collective action. We understand that narrative and transformation, global and local, personal and collective, are articulated on the same stage.

The decolonial turn presents a double challenge for narrative research. On the one hand it resignifies knowledge, methodologies, the academic context and the meaning of narrative as a decolonizing process; and on the other hand, it places us in the field of life to co-construct, in the social and educational context, spaces of shared knowledge and joint action, with performative methodologies that provoke an alternative social and educational debate.

Keywords: social transformation; epistemologies; horizontal methodologies

-¿Los elfos pintan? -preguntó Clara.

-Si-dijo Petrus-, caligrafían y pintan, pero sólo lo que tienen delante. También cantan o escriben poemas para conmover el alma, y lo hacen muy bien, la verdad. Pero eso no basta para cambiar la realidad. -¿Qué hace falta para cambiar la realidad? -Pues historias-dijo

(Barbery, 2015)

\section{PRESENTACIÓN}

En este artículo nos interesa desarrollar una visión de la narrativa que entronca con lo que se viene denominando "Giro decolonial” (Castro-Gómez y Grosfoguel, 2007a; Rivas 2020a). Esto es, pensamos que nuestra perspectiva acerca de la indagación narrativa es convergente con los postulados decoloniales desarrollados por el movimiento modernidad/colonialidad o por perspectivas similares que se vienen desarrollando desde realidades socio-políticas diferentes, como es el caso de Boaventura de Sousa Santos (2017, 2019), Joâo Paraskeva (2018 y 2019) o, desde otra perspectiva, Marta Nussbaum (2005). Entendemos que una orientación narrativa, ya sea en el campo de la investigación, la docencia o la acción social, necesariamente conlleva una transformación en el contexto en el que se construye, en el sentido que ya hemos explicado. Por tanto narración y transformación social deberían ser términos de un mismo campo semántico (si se nos permite la licencia). Toda indagación narrativa, siguiendo a Clandinin (2013) supone un nuevo relato (re-telling) que implica la reconstrucción de la acción (re-living). En este sentido planteamos que se puede hacer una lectura decolonial de esta perspectiva.

Vamos a plantear en primer lugar, una mirada política de la indagación narrativa, a modo de puesta en escena del desarrollo que pretendemos en este trabajo. El escenario actual de la investigación educativa, como en cualquier otra etapa de la historia, está fuertemente intrincado con las políticas públicas, por lo que se hace necesario un breve análisis de cómo es este vínculo. A continuación, como segundo apartado, nos planteamos cómo los postulados de la pedagogía en el escenario actual, neoliberal, burocrático, gerencial y protocolizado, representan una narrativa singular de lo educativo, que está colonizando el terreno de la cotidianidad de los diferentes contextos de la educación. El debate sobre las políticas públicas educativas se está haciendo desde 


\section{E S T U D I O Y E N SAYOS}

un relato particular que anula cualquier posibilidad de transformación al limitar las fronteras de la acción pedagógica a los ámbitos contables de una sociedad mercantilizada. En tercer lugar, a partir de esta premisa ponemos en cuestión elementos relevantes de este relato en tanto que "subalterniza" a los sujetos, especialmente de los colectivos marginados socialmente, debido a que se desarrolla desde una relación específica de poder que se propone como inherente a la relación educativa. Por último planteamos algunas claves de cómo entendemos la indagación narrativa como proceso decolonizador, poniendo en evidencia prácticas y metodologías diferentes que generalmente presentan un carácter performativo (Fernández, Leite y Márquez, 2019).

\section{LA NARRATIVA COMO ACCIÓN POLÍTICA}

Podemos tener la tentación, desde la perspectiva utilitarista hegemónica de la investigación que estamos viviendo, de pensar que hablar de investigación (o indagación) narrativa es hablar de una estrategia de investigación que toma en cuenta los relatos de los sujetos para extraer la información necesaria para elaborar interpretaciones por parte de los investigadores e investigadoras a cargo. Esta perspectiva no dejaría de situarse en una posición de corte positivista, más centrada en el dato que en el sentido, en el investigador que en el participante, y en el conocimiento válido (y validado) antes que en la construcción compartida de la realidad. Desde nuestra perspectiva, cuando hablamos de indagación narrativa nos situamos ante un posicionamiento epistemológico, político, ideológico y moral. Esto es, una forma de comprender el conocimiento y sus procesos de construcción; una idea acerca de la participación en lo colectivo y la construcción de una sociedad democrática; un sentido de la historia, del sujeto y del mundo; y, por último, un proyecto de futuro de acuerdo a modelos de convivencia basados en la pluralidad, la diversidad y la solidaridad.

Teniendo en cuenta estos principios, entendemos que no todo lo que se etiqueta como narrativo en el paisaje actual de la investigación educativa necesariamente participa de este planteamiento. Tal como Rivas (2020a) ha planteado recientemente, hay una fuerte dependencia respecto a los modelos hegemónicos propios del marco neoliberal en el que nos encontramos, que sesga las diferentes perspectivas de investigación de acuerdo a las exigencias de un mercado cognitivo fuertemente regulado y controlado. El debate epistemológico y político necesario para avanzar en un proceso de transformación y de cambio social y educativo ha sido suplido por un utilitarismo metodológico al servicio de intereses diversos. En cualquier caso se coloca al margen de los sujetos, independientemente de la buena voluntad de los y las investigadoras. El proceso resultante se traduce en el control de los resultados de estas investigaciones en tanto que vienen regulados por protocolos establecidos. Podemos decir que los fines no son independientes de los procedimientos, por lo que estableciendo la regulación de estos últimos estamos controlando también sus productos.

La forma como afrontamos la investigación y, en particular, como entendemos la indagación narrativa quiere desvincularse de estos procesos, colocando en primer plano, precisamente, las cuestiones de sentido vinculadas con el sujeto, el conocimiento, la sociedad, las relaciones, etc. Entendemos que la investigación, al igual que la enseñanza y los procesos de formación, se mueven de acuerdo a un planteamiento radicalmente antropológico vinculado con nuestra capacidad de ser parte del mundo en el que vivimos: la comprensión del mundo por parte de los suje- 


\section{E S T U D I O S Y E N A Y O S}

tos y los colectivos y su capacidad de actuar en ellos para su transformación (González, Leite y Rivas, 2018; Rivas, Leite y Cortés, 2017). Esto es, nos conformamos como humanos de acuerdo a nuestra capacidad de dar sentido a cada realidad particular tanto desde un punto de vista colectivo, como de la apropiación singular por parte de cada sujeto y la posibilidad de intervenir en la construcción de nuestro futuro.

No obstante, una de las fuentes de la investigación narrativa suele ubicarse en los movimientos sociales, especialmente en el feminismo de los años 70 y 80 , que reivindicaban una voz propia, diferente de la masculina hegemónica y dominante. Desde estas posiciones se plantea la necesidad de generar nuevas formas de saber, de situarse en el mundo y de construir una relación con la sociedad en todas sus dimensiones. El punto de partida que supuso el conocido lema atribuido a Carol Hanish (1970) "lo personal es político" marca la entrada en el debate público de dimensiones negadas por el relato oficial de la ciencia, poniendo en marcha, junto con otras propuestas parecidas, el conocido como "giro subjetivo" y la reivindicación de la experiencia y las emociones como fuente de conocimiento. Como plantea Hernández (2011), "la escritura personal o subjetiva que caracteriza la perspectiva narrativa de investigación, refleja los valores de un proyecto teórico feminista que defiende la deconstrucción de convenciones académicas e intelectuales excluyentes y cientificistas" (p. 16). Paralelamente tienen lugar posicionamientos parecidos desde la militancia de los grupos minoritarios étnicos y culturales, que sientan las bases de una indagación narrativa de corte político y de transformación social.

Los relatos que elaboramos sobre cómo entendemos nuestra experiencia es, justamente, el modo como construimos la realidad en la que vivimos. Nos sitúan ante la posibilidad de actuar, ya que suponen un conocimiento acerca de la misma (Márquez, Leite y Calvo, 2017). Como plantea Clandinin (2013), vivimos a través de las historias que nos dan cuenta de la experiencia, de la realidad y de los contextos en los que vivimos. Historias que son personales, pero también sociales e institucionales (Márquez, Cortés, Leite y Espinosa, 2019). Esto significa que cada sujeto es portador de un conocimiento del mundo y de la sociedad, el cual es el modo como define su acción y su participación en sus contextos. Este es fruto de su interpretación de su experiencia de los mismos, por tanto de sus tradiciones, de sus historias personales y de la posición que ocupa en ellos. Los diversos colectivos han construido históricamente, diferentes marcos de interpretación que han permitido la apropiación singular de cada sujeto. Hablamos por tanto, de un conocimiento caracterizado por la diversidad, la diferencia y la complejidad, dentro de un marco de comprensión compartido en que esta variedad de relatos tiene sentido. Tal como lo define Walsh (2007, p. 53), se mantiene la unidad en la diversidad, en lo que califica como una lógica inter-epistemológica, clave para la construcción de una nueva democracia.

La indagación narrativa consiste, precisamente, en establecer un diálogo horizontal y democrático entre los diferentes relatos que los ponga en cuestión de cara a avanzar hacia un conocimiento más complejo y diverso (Márquez, Cortés, Leite y Espinosa, 2019). No se trata de llegar a un consenso o una intersección de las diferentes narraciones, sino de propiciar una visión más abierta y diversa del mundo, que permita la convivencia de posiciones distintas en pro de un objetivo común, la transformación del mundo en un sistema más justo y solidario. Lo que la propia Walsh (2007, p. 50) define como pensamiento oposicional, transformador y contrahegemónico. A su vez, este diálogo colectivo supone también una práctica, ya que construye un relato diferente que 
construye una realidad "otra". Como decíamos, este nuevo relato ha sido elaborado en un proceso de diálogo y discusión democrática, lo que nos permite aventurar una realidad igualmente democrática, basada en los principios de equidad, emancipación y solidaridad. De este modo, "es posible hablar de una realidad distinta fruto de una narrativa "otra”, a partir de principios ideológicos, epistemológicos diferentes, que rompan con las hegemonías actuales, del patriarcado, el capitalismo y el pensamiento occidental, positivista y de clase” (Rivas, 2020b).

\section{NARRATIVA Y PEDAGOGÍA. LA COLONIZACIÓN DE UN DISCURSO EDUCATIVO}

Resulta relevante reflexionar acerca de qué se investiga en educación y quién, o qué dinámicas socio-políticas determinan estos focos. No es ingenuo pensar que van emergiendo "temas" sobre los que investigar provocados por intereses diversos y complejos, casi siempre vinculados a dinámicas políticas y/o mercantiles (Rivas, 2008). Sin entrar en detalle en este tema, es fácil comprobar cómo las investigaciones van emergiendo en consonancia con propuestas a menudo ajenas al propio mundo educativo. ¿A quién sirven estas investigaciones y las políticas públicas que las financian y apoyan? ¿Suponen una posibilidad de mejora para los actores educativos o se convierten en losas bajo cuyo peso sucumben las necesidades de cambio de las instituciones educativas? No es baladí la pregunta cuando comprobamos en la producción literaria pedagógica un fuerte énfasis en protocolos, propuestas metodológicas, o fórmulas que garantizan el éxito educativo y profesional. El “cómo hacer" se impone al "qué sentido tiene”, eliminando de esta forma la posibilidad de un debate de tipo epistemológico, político e ideológico sobre el para qué de la educación.

En este debate podrían tener cierto sentido algunas perspectivas críticas “anti-pedagógicas”, provocadas por excesos de este tipo (Fernández-Liria, García y Galindo, 2017). Si bien, tampoco están aportando nada relevante a la discusión más allá de la reivindicación de prácticas caducas e igualmente colonizadas por otras epistemologías poco dadas a la transformación de la educación. En cualquier caso el resultado es el mismo: son propuestas encaminadas a la colonización de la práctica educativa. Esto tiene lugar básicamente por dos procesos: por un lado, a través de esta regulación desde la academia hegemónica y, por otro lado, por una ideología que segrega la teoría de la práctica, eliminando por tanto el debate sobre el sentido. Desde esta perspectiva lo pedagógico se convierte en una narrativa particular de la educación orientada hacia la regulación y el control de la práctica, por tanto, co-responsable de la construcción de una realidad singular en educación, que en este momento podemos calificar de hegemónica y dominante. MaldonadoTorres (2006) identifica en este sentido lo que denomina una "Moralidad maestra" (Master morality), de dominación y control en el corazón de la modernidad occidental, a la que nosotros en otros momentos hemos calificado de “moral neoliberal” (Rivas, Cortés y Márquez, 2018).

A través de esta construcción singular del relato pedagógico se trasluce una epistemología que podemos calificar como gerencialista y burocrática, que se encuentra en el corazón del debate educativo actual. Como De Sousa (2019) plantea, "la política dominante se vuelve epistemológica cuando es capaz de defender activamente que el único conocimiento válido que existe es el que ratifica su propia supremacía” (p. 11). De alguna forma esta epistemología ha construido un "campo semántico" propio sobre ejes como la calidad, la excelencia, el éxito, el esfuerzo, el resultado, la clasificación, etc. (Rivas, Leite y Cortés, 2011). De tal forma que escenifica un mapa de pensamiento que coloniza el espacio educativo e institucional resignificando conceptos y len- 


\section{E S T U D I O Y E N SAYOS}

guajes generados desde propuestas supuestamente alternativas y críticas. En este sentido Walsh (2007) pone en cuestión la validez de términos como “inclusión” para construir una práctica educativa diferente, en tanto que esta es planteada desde una posición de poder, y una jerarquía implícita entre una normalidad regulada, como objetivo común para todas y todos, por tanto sin consideración de la diferencia como condición desde un diálogo horizontal. Lo cual, a la postre, deviene en excluyente. Como plantea Beverley (2013), el cuestionamiento de las narrativas basadas en el criterio de la autoridad, bien sea política, académica, o de cualquier orden, se basa en la "sospecha de que los intelectuales y las prácticas de escritura son, en sí mismas, cómplices para mantener las relaciones de dominación y subalternidad” (p. 355).

Se hace necesario, pues, desde nuestro punto de vista, una decolonización del discurso pedagógico y del relato hegemónico educativo que conflictúe las posiciones de poder desde las que se elaboran. No se trata de buscar un lugar para el pensamiento alternativo, dentro de los marcos de la epistemología colonizadora, tal como se nos muestra demasiado a menudo. Más bien, supone otro modo de hacer, dar nombre, reconceptualizar, reconocer desde la horizontalidad y el proceso de decolonización de pensar-se, pensar-nos, conocer-se, conocer-nos desde categorías propias, no importadas y sutilmente impuestas. Decolonizar, por lo tanto, es un ejercicio, es un hacer, reescribiendo la historia, cuidando la relación, participando en el sentido y los significados conjuntamente (Rivas, Márquez, García y Calvo, 2020). Podríamos hablar, como propone Conle (2001) de una racionalidad del diálogo y la acción comunicativa, orientadas hacia la comprensión y no tanto hacia el éxito de un punto de vista propio. Lo cual, desde una perspectiva decolonial, nos conduce a un relato conflictivo de la realidad, que evidencia las relaciones de poder y dominación implícitas en la epistemología occidental colonial. En este sentido Abdi (2012) pone en evidencia las filosofías de la educación de las así llamadas democracias liberales y sus dinámicas de colonización de los capitales culturales de las minorías diferentes. Algo que se acentúa más en un sistema-mundo cada vez más interconectado, también desde la permeabilidad de sus fronteras.

Un relato decolonial de la pedagogía, por tanto, debería ir encaminado a examinar y transformar las relaciones desiguales de poder, y dirigirse a la transformación estructural sociohistórica. Se plantea la necesidad de reconocer y visibilizar los conflictos socializadores entre distintos grupos a partir del diálogo entre el conocimiento propio con otros conocimientos desde una racionalidad crítica orientada hacia un proyecto de transformación. En este sentido apuntan otras formas de entender el currículum y el desarrollo educativo, basadas en los procesos de comprensión y problematización de las epistemologías hegemónicas, tal como apunta Paraskeva (2018) a través de su idea de currículum itinerante, derivado del desarrollo de la idea de epistemicidio propuesta por De Sousa (2017). Al igual que hace Pinar (2015) con su propuesta de currículum como conversación complicada, en la que presenta la necesidad de incorporar visiones y posiciones alternativas personales y colectivas que problematizan las epistemologías hegemónicas occidentales, patriarcales, capitalistas y supremacistas. Todas ellas suponen construcciones narrativas diferentes que cuestionan las relaciones con el conocimiento y crean espacios de libertad subjetiva en el proceso de construcción de sentido, que debería estar en el foco del proceso educativo.

Una pedagogía con perspectiva decolonial, por tanto, tendría como objetivo permitir que los diferentes grupos y colectivos, especialmente los oprimidos, representen el mundo desde sus 


\section{E S T U D I O Y E N SAYOS}

propias narraciones y lo puedan presentar como propio y en sus propios términos, pues como afirma De Sousa (2019), en relación a las Epistemologías del Sur, "sólo de este modo serán capaces de transformarlo de acuerdo a sus propias aspiraciones” (p. 38). Esto implica formas diferentes de afrontar lo educativo, desde una praxis diferente. En definitiva, como ya hemos planteado, los procedimientos definen las finalidades de la acción, de tal forma que no es posible pensar en una práctica emancipadora de la educación sino hay procedimientos igualmente emancipadores. Lo cual cuestiona las relaciones de poder en educación, las jerarquías establecidas entre estamentos y las epistemologías normalizadas desde lo institucional, como las únicas y verdaderas. Los criterios de verdad, certeza, razón, etc. son revisados y resignificados a partir de una construcción del relato pedagógico diferente.

El concepto de heterarquía, que proponen Castro-Gómez y Grosfoguel (2007b) puede resultar relevante en este sentido ya que re-estructura los sistemas de poder desde una perspectiva de dispositivos heterónomos vinculados en red. Esto es, desde esta perspectiva no existe un nivel básico que gobierna sobre los demás, sino que todos los niveles ejercen algún grado de influencia mutua en diferentes aspectos particulares y atendiendo a sus propias trayectorias biográficas, históricas y experienciales. De esta forma una nueva narrativa en educación necesita estructurarse desde principios democráticos radicales y el respeto a las diferentes epistemologías como formas de conocimiento válidas, a partir de las cuales avanzar en una nueva epistemología más compleja, heterogénea y múltiple, con diferentes temporalidades dentro de un mismo marco de comprensión. Lo cual implica, de acuerdo con Mignolo (2010), una narrativa que desvele las historias coloniales, las relaciones y las diferencias en las historias locales, justo en un contexto en que han sido tradicionalmente negadas o ignoradas. El giro decolonial para una nueva narrativa pedagógica tiene sentido en tanto que supone repensar y reconstruir los ámbitos educativos como espacios de saberes compartidos y escenarios de pensamiento conjunto. Esto significa compartir espacios entre los saberes académicos, producidos por la ciencia, la universidad y la academia, y los saberes legos, populares y diversos que circulan por la sociedad (De Sousa, 2007). Esto implica no solo una forma de reconocimiento de estas otras epistemologías, sino también la posibilidad de un diálogo en igualdad. Como plantea De Sousa (2017), esta ruptura epistemológica implica que "no existe justicia social global sin justicia cognitiva global” (p. 175).

Necesitamos, pues, reconstruir la narrativa pedagógica con una perspectiva decolonial, que rompa con las lógicas gerenciales hegemónicas planteadas y se abra a un diálogo crítico con la incorporación de perspectivas actualmente marginadas, oprimidas, olvidadas o negadas. Y en este sentido asumimos la propuesta de los tres ejes en los que estructura la lógica de la colonialidad (Castro-Gómez y Grofoguel, 2007): colonialidad del poder, colonialidad del saber y colonialidad del ser. En términos del debate educativo estaríamos planteando una nueva narrativa en términos de metodología (Cortés, Leite, Prados y González, 2020), a partir de sistemas de relación diferentes, no autoritarios, democráticos y dialógicos, junto con epistemologías diversas y otros modos de cotidianidad y de experiencia que tome en cuenta el cuerpo como espacio biográfico existencial, la afectividad y la alteridad, como formas constitutivas del sujeto. Todas ellas analizadas desde una perspectiva intersticial trabajando conjuntamente para una pedagogía “otra" que colabore en la construcción del mundo desde una posición radical democrática, equitativa, emancipatoria y solidaria. 


\section{SUJETOS Y VOCES. UNA PERSPECTIVA POLÍTICA DE LA CONSTRUCCIÓN NARRATIVA}

Desde el ya clásico artículo de Hargreaves (1996) "a vueltas con la voz" este ha sido un tema relevante en el debate de la investigación cualitativa, que cuestiona el rol espoliador del investigador, en el contexto de la producción científica y académica. Sin entrar en consideraciones sobre el impacto real de su llamado, lo cierto es que desde la perspectiva que estamos manejando este es un tema central en la configuración de los procesos de investigación. Especialmente cobra más relevancia cuando nos estamos refiriendo a sujetos que han estado tradicionalmente silenciados u olvidados, tal como ya hemos comentado. Normalmente son las voces "autorizadas" (investigadores, intelectuales o docentes, normalmente masculinas) las que construyen el discurso educativo, relegando las historias de los otros sujetos a un lugar irrelevante, o simplemente negado. La dinámica de la investigación, ya sea del tipo que sea, normalmente coloca al sujeto en el lugar de informante, esto es, el que ofrece sus datos para que la persona investigadora elabore sus interpretaciones y construya conocimiento sobre sus voces. En el caso de la enseñanza la voz del sujeto discente no deja de ser sino el eco de la voz autorizada, la del docente, que a su vez también es eco de otras voces legitimadas para elaborar la narrativa válida en una escala jerárquica de poder y legitimación. Ambos se ven obligados a repetir un conocimiento elaborado y validado desde instancias políticas y académicas. Sus relatos de mundo, por tanto sus epistemologías, quedan anuladas.

Resulta pertinente en este caso la pregunta de Tuider (2012) acerca de "quién es el otro" en la investigación, que nos mete de lleno en el debate sobre la alteridad, sin duda uno de los focos relevantes en la perspectiva decolonial y en buena parte de las reflexiones de las ciencias sociales y humanas. "Las preguntas decisivas de los estudios poscoloniales son: ¿quién realmente, con qué objetivo y con qué poder, sobre qué y cómo se habla?” (Tuider, 2012, p. 112). Ponemos en cuestión, de este modo, cómo se construye conocimiento sobre los otros y, como consecuencia, quién está legitimado a hacerlo, más allá del propio sujeto “otro”, revisando estrategias tradicionalmente utilizadas por la etnografía o la antropología o la propia investigación de corte interpretativo. Normalmente se ha considerado una obviedad el derecho del investigador a hacer uso de la voz de los sujetos, convirtiéndose en intérprete, portavoz, traductor, etc. En el mejor de los casos esto se consigue mediante procesos de negociación que autorizan a apropiarse de la voz, de acuerdo a los intereses y finalidades propias del sujeto investigador o investigadora, obviando las relaciones de poder inherentes a estas dinámicas. En la institucionalidad de los procesos de investigación vigente este investigador o investigadora es el único autorizado a hablar, legitimando de esta forma una epistemología colonial, en tanto que proviene de una relación asimétrica, vinculada a los modelos de pensamiento hegemónicos.

Resulta, en este sentido, altamente pertinente, el cuestionamiento ya clásico que hace Spivak (1988) en relación a este tema: ¿Pueden hablar los subalternos? En definitiva, podemos pensar en que los marginados, los excluidos y excluidas, en definitiva, los sujetos “objetos” de investigación, ¿pueden ofrecer sus propias voces, sin el filtro de los investigadores? ¿Qué sería "ser" investigador o investigadora en este escenario? No es posible, desde este punto de vista, pensar en una posición sin intencionalidad, sesgada por las propias construcciones de verdad de cada sujeto que media en las narraciones de los distintos sujetos. Por tanto, si la voz es manejada exclusivamente por uno de los pares en la relación investigador-investigado, son los intereses y finalidades del primero las que obtienen representación y, por tanto, reconocimiento en la construcción 


\section{E S T U D I O Y E N SAYOS}

del pensamiento colectivo. En este proceso ¿dónde se reconoce la posición de sujeto subalterno, dominado, excluido, en la lucha histórica por la transformación social y la emancipación de los colectivos y de los individuos? Nos enfrentamos no solo a un problema metodológico sino esencialmente a uno de tipo ético-político, que golpea, a nuestro entender, en la línea de flotación de la academia y de las instituciones de investigación.

La tradición feminista especialmente vinculada con mujeres de minorías étnicas y culturales, en ese sentido, ha sido pionera en esta reivindicación, desde una posición que asume no solo la complejidad, sino también la perspectiva interseccional que provoca la necesidad de pensarnos desde la multiplicidad que nos caracteriza. Los estudios feministas decoloniales, como los de Moraga y Anzaldúa (2002), dejan claro que no puede nadie representar a nadie si no es en un camino conjunto de descolonización. No deja de ser especialmente crítico este punto de vista, en el que las mujeres no-occidentales cuestionan el sistema se valores de las mujeres occidentales y el modo como han sido cosificadas por estudios coloniales. Una de las aportaciones del giro decolonial es el de indagar desde los márgenes, desde las fronteras, desde espacios de intersección sin una categoría cerrada, que tal modo que ser mujer, indígena, rural y ecologista no puede ser interpretado desde un solo esquema de valores, sino que hay una construcción compartida en la que este conjunto de dimensiones son partes constituyentes.

Hay un profundo sentido político y transformador en este planteamiento ya que pone en cuestión las relaciones de poder implícitas en la subalternidad. Beverly (2013) reflexiona acerca de esta cuestión en relación al Testimonio de Rigoberta Menchú “I, Rigoberta Menchú”. Por un lado, asumiendo su propio testimonio como vehículo en un lugar de autoridad cultural que relega su posición de subalterno, que podría modificar la pregunta de Spivak hacia un formato más propositivo: "Si el subalterno pudiera hablar -es decir, hablar de una forma que en verdad nos importe, que nos haga sentir obligados a escuchar- entonces no sería subalterno" (Beverly, 2013, p. 350). Esto es, el relato es, en sí mismo, un acto transformador que modifica la relación del sujeto con el mundo y su entorno. Esto es así, en tanto que el propio testimonio, la narrativa que se construye, contiene una agenda propia que desarrolla los intereses de su colectivo por hacerse ver y afrontar los cambios que necesita en su proceso de emancipación. Como afirma más adelante este mismo autor, "lo que el testimonio nos obliga a confrontar no es sólo el subalterno como una víctima (auto)representada, sino también como un agente -en el acto mismo de representación-de un proyecto transformativo” (Beverly, 2013, p. 354).

En un sentido parecido, aunque con matices relevantes, Nussbaum (2005) pone en el eje de la construcción de una nueva ciudadanía esta posibilidad que nos ofrece la narrativa de hacernos ver las vidas de quienes son diferentes a nosotros desde una posición de compromiso y entendimiento receptivos y "con ira ante la forma en que nuestra sociedad rehúsa a algunos la visibilidad” (p. 121). De esta forma podemos ver cuanto tenemos de proyectos y metas compartidas y cuáles son las circunstancias que condicionan las posibilidades reales de las personas hacia la acción. Esto nos coloca, como investigadores e investigadoras, en una fuerte contradicción con nuestra posición de privilegio y de autoridad de la que nos dota nuestra posición institucional. De hecho, cuestiona nuestra autoridad para apropiarnos de otras voces de otra forma que no sea a partir del espacio de diálogo y comprensión mutua que venimos proponiendo a lo largo de este trabajo. 


\section{E S T U D I S Y E N S Y O S}

Lo narrativo se convierte de este modo en performativo en tanto que no se limita solo a un discurso simplemente descriptivo o denotativo (Beverly, 2013, p. 352), sino que pone en evidencia el conjunto de procesos y experiencias de marginación y dominación vividas en contextos y escenarios singulares. Por tanto no solo afecta al sujeto que elabora su voz en un acto político de reconocimiento y emancipación, sino que pone en juego el conjunto de la comunidad que comparte estos procesos y en aquellos que provoca resonancias y complicidades. Como propone Alexander (2013), "los practicantes de la etnografía performativa reconocen el hecho de que la cultura viaja en las historias, las prácticas y los deseos de quienes se comprometen con ello" (p. 94) implicando, por tanto, a los individuos como sujetos históricos y transformadores de su realidad a través de sus narraciones y las acciones con las que se construyen.

Esta perspectiva no significa que haya una finalidad esencialista y reivindicativa de las culturas contenidas en las voces subalternas, como alternativas al relato oficial y diferente a él. En primer lugar, porque no existe "pureza" en las narraciones, en el sentido de que den cuenta de una realidad uniforme y diferenciada; lo que podríamos calificar como "autenticidad cultural". Antes bien, cada relato, como apuntaba Freire (2002), contiene tanto al dominado como al dominador, que conviven en cada sujeto, en tanto que estamos compartiendo un espacio social y político, aunque desde posiciones diferentes. En segundo lugar, porque el foco se coloca en la construcción de un conocimiento intersticial en el que nos ubicamos a través del diálogo comprometido y transformador. Lo cual nos lleva plantear, junto con Castro-Gómez y Grosfoguel (2007) una "complicidad subversiva" con el sistema, entendida como una resistencia semiótica capaz de resignificar las formas hegemónicas de conocimiento desde el punto de vista de las epistemes propias de las subjetividades subalternas. Lo narrativo-performativo ubica las voces de los sujetos en un contexto de transformación no desde la (auto)exclusión, sino de la construcción de un nuevo relato capaz de crear realidades nuevas. En este sentido Nussbaum (2005) plantea un nuevo relato en el que de dar cabida en nuestra mente a sujetos, conocimientos y prácticas que nos puedan parecer extrañas, e incluso aterradoras, demostrando "una capacidad de apertura y sensibilidad hacia los demás que corre a contrapelo de muchos estereotipos culturales de autosuficiencia” (p. 132)

Una posición semejante es la que plantea De Sousa (2017) con su concepto de cosmoplitismo insurgente subalterno. Esto es, "la aspiración de los pueblos oprimidos a organizar su resistencia y consolidar coaliciones políticas a la misma escala que los opresores emplean para oprimirlos, es decir, la escala global” (p. 177). Se plantea como una forma de resistencia al pensamiento abisal que no implica uniformidad (aspiración propia de la modernidad occidental, patriarcal y capitalista), sino un proceso de emancipación social que da igual valor al principio de igualdad que al principio de reconocimiento de la diferencia trascendiendo las fronteras de lo local y de lo singular.

\section{EL PROCESO DECOLONIZADOR DE LA NARRATIVA}

Recuperando el punto en que dejamos el apartado anterior, partimos de una posición respecto a la investigación narrativa que se diferencia claramente de otras formas coloniales de investigar. El interés de partir de las voces subalternas en su proceso de reconocimiento y acción transformadora no es obtener la "verdad", cualquier cosa que podamos pensar que es esta, sino promover los intereses del grupo y de las clases sociales que sus narrativas representan (Beverly, 2013), desplazando 


\section{E S T U D I O S Y E N S Y O S}

la centralidad de los intelectuales y lo que estos representan. Cerramos este trabajo recogiendo los planteamientos anteriores para ofrecer una perspectiva decolonial de la investigación narrativa, a partir de esta mirada ético-política que venimos defendiendo. No obstante, la propuesta de una epistemología “otra”, desde la perspectiva decolonial, se sustenta en buena parte en la construcción de unas narrativas "otras” que abran el camino para una acción transformadora. En definitiva, nos movemos en una perspectiva performativa en la que forma y contenido, por así decir, se identifican, generando una unión de pensamiento y acción que supera viejas dualidades.

Nos parece relevante en este sentido el concepto de performance que nos ofrece Butler (2007), como la acción de actuar desde el discurso, vinculando, de este modo, el relato con la transformación. Cuando esta autora habla de discurso, hace hincapié en que las actividades están determinadas discursivamente y solo viven performativamente, en su acción localizada por parte de los agentes. Hablamos, por tanto, de una acción situada, que nos aleja de la uniformidad y aparente universalidad de los protocolos estandarizados de investigación, por tanto que no puede reproducirse de forma automática y estrictamente pautada, por lo que incorpora de forma inherente la posibilidad de la transformación social, tal como estamos viendo. Denzin (2001) retoma a su vez esta posición en la búsqueda de una nueva forma interpretativa, donde los instrumentos y los métodos adquieren un sentido performativo, donde se cruzan las diferentes dimensiones del sujeto, con valor intersticial, con el objetivo de crear textos que dan poder, criticando el modo como está organizado el mundo y proponiendo formas alternativas en que este podría ser organizado (O’Neil, Roberts y Sparkes, 2015). Lo que venimos planteando nos acerca a la perspectiva decolonial y su propuesta de transformación a partir de una epistemología "otra" situada en las voces de los sujetos subalternos.

Los planteamientos de Tuider (2012), ya comentados, nos ayudan también a situarnos en esta perspectiva en tanto que está poniendo el foco en el sentido de las narrativas desde el discurso que se elabora o construye y el conjunto de ideas, representaciones y tramas que forman parte de las mismas. En tanto que pone en relación la indagación biográfica y el análisis del discurso, es posible analizar las narrativas como discursos configurados, pero también como configuradores, reguladores, estructuradores de sentidos, valores, formas de ser, actuar, sentir, etc., conforme a culturas particulares. Indagar narrativamente no solo da cuenta de una historia sino que pone al descubierto las prácticas que la han configurado y las dinámicas sociales, políticas, económicas y de género que han participado en las mismas. Riessman (2008) se refiere a este proceso como análisis dialógico performativo, avanzando en el mismo principio el carácter transformador y ligado a la acción de la construcción narrativa y biográfica vinculado a sujetos singulares de colectivos en situaciones de dependencia.

En definitiva estos diferentes planteamientos apuntan a una investigación "otra” que plantea otras formas de hacer, de relacionarse y de conocer, que incorporan en sus prácticas los principios ético-políticos de una sociedad diferente, basada en los principios de equidad, libertad y solidaridad. Desde la perspectiva biográfico-narrativa no son pocas las propuestas que incorporan un planteamiento emancipador y radicalmente democrático en sus modos de hacer, pensar y relacionarse (O 'Neill, Roberts y Sparkes, 2015) a partir de la idea de diálogo narrativo, biografías participativas y prácticas performativas (Cortés, Leite, Márquez y González, 2020).

Resulta relevante en el ámbito de la práctica de investigación las reservas que muestran investigadores e investigadoras provenientes de culturas minoritarias, ya que tradicionalmente 


\section{EST U D I O Y E N SAYOS}

han sido objeto de estudio desde prácticas cosificadoras y de dominación. Tahiwai Smith (2016) es clara en su rechazo a estas prácticas cuando plantea la devastada historia que la investigación ha tenido en las poblaciones indígenas de todo el mundo. La investigación ha significado una relación de poder completamente asimétrica por parte del investigador y la epistemología etnocentrista que le configura. Con estos antecedentes nombrar la palabra investigación entre poblaciones y grupos subalternos ha supuesto una gran desconfianza y ofensa. Esta llamada de atención nos alerta acerca de lo que ha significado investigar desde un espacio académico. Esta creencia aún sigue arraigada, mediante el control de estrategias metodológicas y sistemáticas apoyadas académicamente haciendo preguntas “apropiadas” y visibilizando un proceso riguroso construido sobre categorías de pensamiento eurocéntricos y patriarcales. Lo cual, como mucho, habla a favor de la buena voluntad de los investigadores e investigadoras, pero poco acerca de la posibilidad de una mejora de la sociedad.

Desde nuestro punto de vista se hace necesario un replanteamiento de lo metodológico que incorpore esta perspectiva performativa en un intento de participar en los procesos de cambio y transformación social. Este pasa por una posición de co-construcción del sentido a partir de dinámicas colaborativas implicadas con los entornos y los sujetos. En este sentido la perspectiva decolonial propone una ciencia centrada en las relaciones sujeto-sujeto que desplace a los participantes al centro en detrimento de la posición de privilegio tradicional del investigador. Se nos invita, de este modo, a conocer el mundo "con" los sujetos subalternos y no asumir su representación, desde una autoridad arrogada que poco tiene que ver con estos. En este sentido Emma León (2005) nos plantea la ética de la responsabilidad en el modo de ser y estar en el mundo de cara a articular una relación cuidadosa con el otro. La pregunta sobre cómo construimos el sujeto participante, relevante en muchos sentidos desde una perspectiva crítica, quizás debería replantearse en cómo nos co-construimos mutuamente sujetos participantes e investigadores. Esto significa una mirada crítica sobre uno mismo, así como sobre el cuidado de la palabra y el modo de nominar y pensar al otro en un universo cultural, replanteando nuestra "sensibilidad, nominación y discursividad” (León, 2015, p. 236). El modo de pensar el otro es también pensar cómo nos vamos construyendo nuestra forma de ver el mundo y cómo lo nombramos; es decir, el uso cuidadoso del lenguaje al nombrar al otro y su universo culturalmente construido. Esta autora nos propone una competencia ontológica, es decir la posibilidad de instalarnos en el lugar del otro y así construir otro modo de relaciones y comprensiones con/el del otro. En otros términos es similar a lo que plantea Nusbbaum (2005) cuando nos habla de la imaginación narrativa.

Avanzar en esta posición nos lleva a cuestionar los procedimientos como protocolos ordenados preestablecidos. Más bien nos adentramos de alguna forma en el terreno de lo desconocido en tanto que no hay un diseño definido que nos marque el camino. Como plantean Morin, Ciurana y Motta (2002), el método es el camino, por lo que se va construyendo en tanto que avanzamos en la dinámica de las relaciones y la construcción compartida en un entorno social singular a partir de vínculos que nos comprometen. En este mismo sentido Ortiz, Arias y Pedrozo (2018) nos proponen pensar en la "no-metodología", que nos permita reinventar los procesos de conocimiento, acercamiento a la realidad y la representación; no tanto por la ausencia de cualquier tipo de guía u orientación, sino en cuanto a la posibilidad de ir generando procedimientos en tanto que transitamos por la investigación. En esta tarea es imprescindible decolonizar el sujeto investigador pero también los procesos, los contextos académicos y el modo de hacer ciencia. 


\section{EST U D I S Y E N SAYOS}

Algo que en la coyuntura actual está fuertemente controlado desde el principio de capitalismo cognitivo que dificulta la puesta en marcha de esta perspectiva.

En este proceso de decolonización de la metodología narrativa podemos hablar del "hacer decolonial" que se enfoca en develar y transformar las relaciones desiguales de poder. El hacer decolonial se concibe como un proceso decolonial en todos los aspectos, desde las relaciones y las acciones, el observar comunal, el conversar alterativo y el reflexionar configurativo. Como afirma Castillo (2017), investigar no significa ir a conocer una realidad sino dejarla venir, convivir con ella de modo que no la interpelamos sino que es ella la que nos interpela. El hacer decolonial no solo pretende revisar la metodología desde esta perspectiva sino que da un paso más al reconocer a los participantes como el otro-yo en un diálogo entre iguales. "Implica vivir en/ por/para la propia comunidad que pretende descolonizarse. Es por ello que todo proceso decolonizante es una autodecolonización" (Ortiz, Arias y Pedrozo, 2018, p. 182). En este sentido nos parece relevante apostar por procesos no solo de co-construcción, sino también co-autoría, que den cuenta de las propias voces de los sujetos y las dinámicas que tienen lugar. Trabajos como el de Márquez, Cortés, Leite y Espinosa (2019), son relevantes en este sentido en tanto que son elaborados conjuntamente contando con una autoría compartida.

En esta línea destacamos algunos espacios en los que detenernos a pensar, no como metodología sino como un hacer decolonial (Ortiz y Arias, 2019; Ortiz, Arias y Pedrozo, 2018), tales como la colaboración, el conversar alterativo y la escucha, y especialmente el sentido de la ética comunitaria, del cuidado, de la responsabilidad y de la liberación en todo el proceso (Dussel, 2007). La indagación narrativa, como venimos diciendo a lo largo de este trabajo, se considera mediadora decolonial que invita a la acción transformadora en todos los sentidos y todos los contextos. En la actualidad autores como Leyva y Speed (2008) aportan al debate metodológico decolonial el concepto de investigación co-labor, a modo de introducir en el debate de metodologías-otras el activismo en el modo de producir conocimiento en el ámbito académico. La perspectiva co-labor plantea emprender una labor colaborativa con una agenda compartida, con un diálogo de intereses, procesos y fines a partir de talleres compartidos con las comunidades y la co-construcción de textos con el reconocimiento de las dos partes, comunidad y academia. Todo el proceso se va tejiendo en un diálogo de saberes horizontal en el que las tensiones son un motivo más para profundizar en el diálogo. A diferencia de hablar de investigación colaborativa Leyva y Speed (2008) dan un giro al concepto al pensar que el término colaborativo puede ser demasiado amplio y lo que aporta la investigación decolonial va mucho más allá, como un caminar que busca descolonizarse en un proyecto colectivo "que genera el cruce de las academias otras, activismos abiertos y movimientos sociales” (Ibidem, 2008, p. 52).

\section{BUSCANDO UN CIERRE}

La indagación narrativa desde una perspectiva decolonial nos sitúa en un planteamiento epistemológico "otro"; es decir, nos platea otras preguntas de partida sobre el mundo, que nos interpelan como personas y como investigadores e investigadoras. En estas preguntas se pone en juego el compromiso con las historias, la sociedad y la educación global y local, desde una perspectiva descolonizadora, es decir, nos situamos en una posición de justicia social con aquellos colectivos 


\section{E S T U D I S Y E N A Y O S}

y personas vulnerables. La narrativa nos invita desde la perspectiva decolonial a co-construir narrativas alternativas desde posiciones alternativas al sentido segregador, tecnocrático, neoliberal y patriarcal de la educación actual. Las preguntas serían ¿qué sentido social y político tiene la indagación narrativa? Nos preguntamos por la relación entre las grandes narrativas y las narrativas locales, situadas en espacios históricos en relación con las vidas cotidianas que ponen en cuestión y desafían un conocimiento hegemónico buscando un espacio que lo cuestione.

Por otra parte la narrativa, desde una perspectiva decolonial, implica co-construir un lugar común de partida en el que nos reconocemos, nos pensamos y nos reconfiguramos en la acción transformadora entrelazada en un espacio ético-político de relaciones, preguntándonos quién es el "otro" en el mundo y en mí. Nos preguntamos ¿quién investiga? ¿quién es el "otro, otra, otros, otras” en mí?. Situándonos en un espacio de transformación personal y social que debe interpelar al contexto social y cultural hacia un debate más amplio de otros modos de ver, sentir y vivir el mundo. Este escenario nos sitúa en un espacio de saberes compartidos en un marco de pensamiento conjunto, que no quiere decir homogéneo, sino diverso. La complejidad y el pensamiento intersticial se ponen en juego superando dualidades y problematizando las epistemologías. En este sentido el relato de las vidas de las personas oprimidas importa y la situación de subalternidad se transforma. En este espacio el dialogo, la dignidad y la horizontalidad sitúan la narrativa en una posibilidad decolonial en el sentido, en el diálogo horizontal y en su finalidad.

Por último, la perspectiva decolonial no solo queda en el sentido epistemológico y práctico transformador, sino también como un medio decolonizador, en lo que llamamos un hacer decolonial, es decir, en el propio proceso de hacer narrativo. Éste es un espacio ético contingente, de co-contrucción, co-interpretación y co-autoría horizontal, que nos invita a formar parte, desde la investigación, de una red diversa, con un mismo marco de compromiso y radicalmente democrática, de co-conocimiento (De Sousa, 2010) y colaboración para otro relato de la educación y la sociedad, mediante acciones/huellas decoloniales que configuran -y se configuran en/desde/ por/para- el sentir-pensar-hacer humano”(Ocaña y Arias, 2019). En este hacer narrativo también nos preguntaríamos ¿cómo hacemos en común?

\section{BIBLIOGRAFÍA}

Abdi, A. A. (2012). Decolonizing Philosophies of Education. Rotterdam: Sense.

Barbery, M. (2015). La vida de los elfos. Barcelona: Seix Barral.

Beverly, J. (2013). Testimonio, subalternidad y autoridad narrativa. En N. Denzin, \& Y. Lincoln (Coords.), Las estrategias de investigacion cualitativa (págs. 343-360). Barcelona: Gedisa.

Butler, J. (2007). El género en disputa. El feminismo y la subversión de la identidad. Barcelona: Paidós.

Castillo, D. (2017). Des-apreciaciones del método científico y la vacante en la metodología decolonial. Hoja Filosófica, 1(43), 5-19.

Castro-Gomez, S., \& Grosfoguel, R. (2007a). Prólogo. Giro decolonial, teoría crítica y pensamiento heterárquico. En S. Castro-Gomez, \& R. Grosfoguel (coords.), El giro decolonial. Reflexiones para una diversidad epistémica más allá del capitalismo global (págs. 9-24). Bogotá: Siglo del Hombre editores. 


\section{E S T U D I S Y E N A Y O S}

Castro-Gómez, S. \& Grosfoguel, R. (coords.). (2007b). El giro decolonial: reflexiones para una diversidad epistémica más allá del capitalismo global. Bogotá: Siglo del Hombre Editores.

Clandinin, D. (2013). Engaging in narrative inquiry. Walnut Creek, CA: Left Coast Press.

Conle, C. (2001). The Rationality of Narrative Inquiry in Research and Professional Development. European Journal of Teacher Education, 24(1), 21-33. https://doi.org/10.1080/02619760120055862

Cortés, P., Leite, A.E, Prados, M.E. y González, B. (2020). Trayectorias y prospectivas metodológicas para la investigación narrativa y biográfica en el ámbito social y educativo. En J. Sancho, F. Hernandez, L. Montero, J. De Pablos, J. Rivas, \& A. Ocaña (coords.), Caminos y derivas para otra investigación educativa y social. Barcelona: Octaedro.

De Sousa Santos, B. (2007). La Universidad en el siglo xxi Para una reforma democrática y emancipatoria de la universidad. La Paz: Plural Editores.

De Sousa Santos, B. (2017). Justicia entre Saberes: Epistemologías del Sur contra el Epistemicidio. Madrid: Morata.

De Sousa Santos, B. (2019). El fin del imperio cognitivo. La afirmación de las epistemologías del sur. Madrid: Trotta.

Denzin, N. (2001). The reflexive interview and a performative social science. Qualitative Research, 1(1), 23-46. https://doi.org/10.1177/146879410100100102

Dussel, E. (2007). Política de la liberación I. Historia mundial y crítica. Madrid: Trotta

Fernández, P., Leite, A. y Márquez M.J.(2019). Narrativas disruptivas en la formación inicial del profesorado. Transformar aprendiendo. Cabás. 22, 61-72. http://eoi.citefactor.org/10.11206/ $\underline{\text { muesca.01.22.006 }}$

Fernandez-Liria, C., Garcia, O., \& Galindo, E. (2017). Escuela o barbarie. Entre el neoliberalismo salvaje y el delirio de la izquierda. Madrid: Akal.

Freire, P. (2002). Pedagogía de la Esperanza. Buenos Aires: Siglo XXI.

González, B., Leite, A. E. y Rivas, J.I. (2018). La indagación educativa como herramienta de transformación social. En V. Martín \& M. T. Castilla (Coords), Educación, derechos humanos y responsabilidad social (pp. 127-142). Barcelona: Octaedro.

Hanish, C. (1970). The Personal is political. En S. Firestone, \& A. Koedt, Notes from de the second year: Women's liberation. Major Writings of the radical feminists (págs. 76-78). New york: Radical Feminism.

Hargreaves, A. (1996). A vueltas con la voz. Kikiriki. Cooperación educativa, 42-43, 28-34.

Hernández, F. (2011). Las historias de vida en el marco del giro narrativo en la investigación en Ciencias Sociales: los desafíos de poner biografías en contexto. En F. Hernandez, J. M. Sancho, \& J. I. Rivas (coords.), Historias de vida en educación. Biografías en contexto (págs. 13-22). Barcelona: ESBRINA RECERCA http://bit.ly/3eCWAhj

León, E. (2005). Sentido ajeno. Competencias ontológicas y otredad. Barcelona: Anthropos. https://doi. org/10.5565/rev/papers.2026

Leyva, S., \& Speed, S. (2008). Hacia la investigación descolonizada: nuestra experiencia de co-labor. En X. Leyva, A. Burguete, \& S. Speed (coords.), Gobernar (en) la diversidad: experiencias indígenas desde América Latina. Hacia la investigación de colabor (págs. 34-59). México D.F.: CIESAS, FLACSO. 


\section{EST U D O S Y E N AYOS}

Maldonado-Torres, N. (2006). Against War. Views from the Underside of Modernity. Durham/London: Duke University Press.

Marquez, M.J., Cortes, P., Leite, A.E., \& Espinosa, I. (2019). Narrativas de vida y educación. Diálogos para el cambio social. Barcelona: Octaedro.

Márquez, M.J., Leite, A.E., y Calvo, P. (2017). Pensarnos a nosotras en el encuentro con los “otros/as”. Investigación narrativa y transformación. Revista del Instituto de Investigaciones en Educación Facultad de Humanidades - UNNE. 10, 1-151. https://doi.org/10.30972/riie.8103655

Mignolo, W. (2010). Historias locales, diseños locales. Madrid: Akal.

Moraga, C. y Anzaldúa, G. (2002). This Bridge Called My Back: Writings by Radical Women of Color. San Francisco: Third Woman Press.

Morin, E., Ciurana, E., \& Motta, R. (2002). Educar en la era planetaria. El pensamiento complejo como método de aprendizaje en el error y la incertidumbre humana. Valladolid: Unesco / Universidad de Valladolid.

Nussbaum, M. C. (2005). El cultivo de la humanidad. Una defensa clásica de la reforma en la educación liberal. Barcelona: Paidós.

O ' Neill, M. R., Roberts, B, y Sparkes, A. C. (coords.) (2015). Advances in Biographical Methods. New York: Routledge.

Ortiz, A. M., Arias, M.I y Pedrozo Z. (2018). Metodología 'otra' en la investigación social, humana y educativa. El hacer decolonial como proceso decolonizante . FAIA, 7(30), 172-200.

Ortiz, A., \& Arias, M. (2019). Altersofía y Hacer Decolonial: epistemología 'otra' y formas 'otras' de conocer y amar. Utopia y Praxis Latinoamericana. 24(85), 89-116.

Paraskeva, J. M. (2018). Against the Epistemicide. Itinerant Curriculum Theory and the Reiteration of an Epistemology of Liberation. En M. Uljens, \& R.M.M. Ylimaki. (coords.), Bridging Educational Leadership, Curriculum Theory and Didaktik: Non-afirmative Theory of Education (págs. 199-213). New York: Springer.

Paraskeva, J. M. (2019). Justicia contra el epistemicidio. Hacia una breve crítica de la razón occidental moderna. Con-Ciencia Social, 3, 157-174. https://doi.org/10.7203/con-cienciasocial.3.16795

Pinar, W. (2015). Educational experience as lived. Knowledge, history, Alterity. New York: Routledge.

Riessman, C. (2008). Narrative methods in the human sciences. New York: Sage.

Rivas, J. I. (2020a). La investigación educativa hoy. Del rol forense a la transformación social. Márgenes, Revista de Educación de la Universidad de Málaga, 1(1), 3-22. https://doi.org/10.24310/mgnmar. $\underline{\text { vli1.7413 }}$

Rivas, J. I. (2020b). Una investigación “otra, para una educación “otra”, para una sociedad "otra. Hegoa. 9, 26-27

Rivas, J.I., Cortés, P., \& Márquez, M. J. (2018). Experiencia y contexto. Formar para transformar. En C. Monge, \& P. Gomez (Coords.), Innovando la docencia desde la formación del profesorado (págs. 109124). Madrid: Síntesis.

Rivas, J.I., Leite, A. E. y Cortés, P. (2011). Luchando contra la historia. Obstáculos para la innovación en la formación del profesorado, derivados de las historias escolares del alumnado. Educación y Pedagogía. 23(61), 67-79 


\section{EST U D O S Y E N AYOS}

Rivas, J.I., Leite, A.E. y Cortés, P. (2017). Deconstruyendo las prácticas de formación: narrativa y conocimiento. En M. Pérez y J. Rodríguez (coords.), Buenas prácticas docentes del profesorado universitario. Barcelona: Octaedro

Rivas, J. I., Márquez, M., García, M., \& Calvo, P. (2020). Perspectiva decolonial de la investigación educativa y social. Rompiendo con la hegemonía epistemológica. En J. Sancho, F. Hernandez, L. Montero, J. De Pablos, J. Rivas, \& A. Ocaña (coords.), Caminos y derivas para otra investigación educativa y social . Barcelona: Octaedro.

Spivak, G. C. (1988). Can the subaltern speak? En C. Nelson, \& L. Grossberg (eds.), Marxism and the Interpretation of Culture (págs. 280-316). Urbana: University of Illinois Press.

Tuhiwai, L. (2016). Descolonizar las metodologías. Investigación y pueblos indígenas. London: LOM.

Tuider, E. (2012). Contando historias/narraciones en un contexto postcolonial. Análisis del discurso y análisis biográfico como métodos horizontales. En S. Corona Berkin, y O. Katlmeier (Coords.), En Diálogo. Metodologías Horizontales en Ciencias sociales (págs. 111-135). Barcelona: Gedisa.

Walsh, C. (2007). Interculturalidad y colonialidad del poder: Un pensamiento y posicionamiento “otro" desde la diferencia colonial. En S. Castro.-Gómez \& R, Grosfoguel. (Eds.), El giro decolonial Reflexiones para una diversidad epistémica más allá del capitalismo global (págs. 47-62). Bogotá: Siglo del Hombre Editores; Universidad Central, Instituto de Estudios Sociales Contemporáneos y Pontifi cia Universidad Javeriana, Instituto. 